\title{
La Blanca, un ASENTAMiento URbano MAYA EN LA CUENCA DEL RÍo MopÁN
}

\author{
La Blanca, a Mayan Urban Settlement in the Mopan River Basin \\ Gaspar Muñoz-Cosme \\ Cristina Vidal-Lorenzo
}

Resumen: El objetivo de este trabajo es presentar los resultados de una investigación realizada recientemente en el asentamiento maya de La Blanca, Guatemala, cuyas características urbanas y arquitectónicas revelan datos significativos de su importancia social y cultural y del papel que jugó en el ámbito de la cuenca del río Mopán. La metodología empleada en esta investigación, así como la puesta en valor de este importante patrimonio cultural, nos ha permitido demostrar cómo, al mismo tiempo, es factible llevar a cabo acciones susceptibles de favorecer el desarrollo social, económico y cultural de las poblaciones vinculadas a sitios arqueológicos como éste.

Palabras clave: arqueología, arquitectura, urbanismo, patrimonio, maya, desarrollo.

Abstract: The purpose of this paper is to discuss about a recent research carried out at La Blanca, an ancient Mayan settlement in Guatemala. The urban and architectural features of this site reveal us important data about its social and cultural significance, and also about the role it played in the Mopan river basin area. The research methodology as well as La Blanca cultural heritage enhancement has produced results that demonstrated the possibility of conducting social action aimed at promoting economic and cultural developing of neighboring communities.

Keywords: archaeology, architecture, urbanism, heritage, Maya, development.

Gaspar Muñoz Cosme, doctor arquitecto por la Universidad Politécnica de Valencia, España. Investigador en el Instituto de Restauración del Patrimonio de la Universidad Politécnica de Valencia, España. Temas de especialización: conservación y restauración del patrimonio arquitectónico y arquitectura precolombina. Correo electrónico: gmcosme@upv.es.

Cristina Vidal Lorenzo, doctora en geografía e historia por la Universidad Complutense de Madrid, España. Investigadora en el Departamento de Historia del Arte de la Universidad de Valencia. Temas de especialización: arte y arqueología maya. Correo electrónico: cristina.vidal@uv.es.
Enviado a dictamen: 17 de junio de 2013.

Aprobación: 06 de agosto de 2013.

Revisiones: 1 . 
L a Blanca es una de las numerosas entidades políticas mayas fundadas en la cuenca del río Mopán en el sureste del Petén guatemalteco. Es éste un territorio de valles fluviales que desde el año 1987 ha sido objeto de un sistemático reconocimiento arqueológico por parte del Proyecto Atlas Arqueológico de Guatemala, dirigido por Juan Pedro Laporte, a partir del cual se pudo establecer que el tipo de organización política predominante en esta región es el de múltiples núcleos urbanos de tamaño restringido y de naturaleza autónoma o descentralizada que convivieron con otros de formación unitaria, es decir, que no incluyen sitios dependientes formados por el proceso de escisión de linajes de parentesco (Laporte, 1996: 40 y ss; 1998: 152). ${ }^{1}$

Entre los sitios de formación unitaria destacan los concentrados en la margen oeste del río Salsipuedes, un territorio de sabana húmeda flanqueado al oeste por una serranía de suaves elevaciones que marca el límite occidental del Valle del Mopán. Entre estos sitios se encuentran La Blanca, Los Lagartos, La Amapola, El Aguacate, Chilonché y Muxanal. Es muy posible que estos centros estuvieran asociados a las grandes ciudades del noreste, como Yaxhá, Nakum, Naranjo o Caracol, y que al mismo tiempo mantuvieran una intensa relación con las entidades segmentarias del noroeste de las Montañas Mayas, sin que haya existido desigualdad cultural entre ellos, pero sí diferencias a nivel de integración política (Laporte y Mejía, 2001; Vidal y Muñoz, 2011). De hecho, en esta misma subcuenca se registraron dos centros de carácter segmentario, El Camalote/Dolores y Salsipuedes, ubicados, curiosamente, en los extremos meridional y septentrional de este territorio (ver mapa 1).

La propuesta de este modelo de organización política para la región sureste de Petén supuso un nuevo enfoque en el controvertido debate acerca de cómo estaban organizados políticamente los antiguos mayas, un modelo que difiere de aquellos más tradicionales defensores de la existencia de grandes ciudades que dominaban amplias zonas periféricas con asentamientos dispersos (Laporte, 2001: 137).

Partiendo de estas premisas, uno de los objetivos del Proyecto La Blanca, el cual lleva realizando investigaciones en este sitio arqueológico desde el año 2004 (Muñoz y Vidal, 2005, 2006; Vidal y Muñoz, 2007), ha sido determinar el papel que jugó La Blanca en una región compleja desde el punto de vista de su organización política, pero muy rica en recursos naturales, y donde las vías fluviales actuaron como valiosas vías de comunicación e intercambio entre las tierras altas y las tierras bajas mayas.

Otras de las preguntas a las que este proyecto pretende dar respuesta son las siguientes: ¿a qué centro mayor del noreste de Petén estaba asociado La Blanca? y icómo hemos de considerarlo: una ciudad, un asentamiento urbano, un núcleo de población?

Empezaremos por la última pregunta. El concepto de "ciudad" en el área maya ha sido y sigue siendo objeto de diversas teorías, posturas y aproximaciones de diversa naturaleza, en parte debido a la inexistencia de un consenso general acerca de la definición de este término y de muchos otros asociados al fenómeno urbano (Taladoire, 2008) y a la dificultad que entraña su aplicación al estudio de sociedades antiguas. Tradicionalmente, uno de los principales indicadores para definir una ciudad ha sido el tamaño o volumen constructivo y la densidad poblacional, si bien estos criterios no se adaptan a la realidad del área maya, donde aún siguen faltando muchos datos para determinar el número de habitantes de un asentamiento y donde la dimensión de los mismos es muy dispar. Tampoco ha contribuido a llegar a un acuerdo el hecho de que la mayoría de las investigaciones arqueológicas se ha concentrado en los grandes centros, dominados por la presencia de edificios y monumentos de carácter público y ritual, que pronto se convirtieron en otros de los elementos de diagnóstico a tener en cuenta a la hora de establecer los criterios definitorios del concepto de ciudad. Sin embargo, muy escasos son los testimonios de los que disponemos acerca de otros indicadores de naturaleza funcional indispensables en el desarrollo de todo centro urbano, tales como mercados, almacenes, baños o talleres (Ciudad e Iglesias, 2001: 12), debido principalmente a la dificultad de documentar en las excavaciones arqueológicas dichas estructuras, por lo general de carácter perecedero. 
Para la región del sureste de Petén, Laporte (2001: 155) se inclina por considerar los núcleos urbanos de este territorio como ciudades debido a la presencia en ellos de una serie de elementos constitutivos de carácter arquitectónico que estima definitorios para tal categoría, y que son: el conjunto de tipo Grupo E, el conjunto de tipo Acrópolis, el desarrollo de calzadas internas y la existencia de terrenos para el Juego de Pelota, si bien sólo 12 sitios de esta área incluyen estos cuatro elementos.

Ante la disparidad de criterios, hemos optado por referirnos a La Blanca como un asentamiento urbano, entendiendo por ello la presencia de una ordenación premeditada de elementos arquitectónicos y de espacios públicos que conforman un conjunto diseñado y construido para albergar una población estructurada y para realizar en dichos espacios todas las actividades de carácter oficial, mercantil, conmemorativo o simbólico.

\section{La Blanca. Arquitectura y urbanismo}

A pesar de su reducido tamaño en comparación con las grandes capitales de reinos poderosos, como Tikal, La Blanca exhibe una arquitectura de gran calidad y notables proporciones, de ahí que la forma más adecuada para describirla sea por los rasgos más característicos que presentan su arquitectura y su estructura urbana que, aunados a los resultados de las investigaciones arqueológicas anteriormente citadas, nos permitirán reconstruir en parte su pasado y su época de mayor esplendor $y$, por consiguiente, dar respuesta a las otras dos cuestiones a las que más arriba hemos hecho referencia.

Estos rasgos más significativos son: la orientación de la trama urbana, el conjunto monumental de carácter religioso (Grupo Sur), la Calzada, la Aguada, los conjuntos residenciales de la población (Grupo Oeste), la Gran Plaza Norte y el conjunto monumental de tipo Acrópolis, unidad de residencia de la élite.

Así, encontramos en primer lugar que la orientación de la trama urbana sigue un eje norte-sur que ordena todo el asentamiento, si bien este eje tiene una apreciable desviación de 12 grados hacia el noroeste.
Esto es habitual en las ciudades mayas, aunque por lo general ese desvío suele ser hacia el noreste.

Del levantamiento topográfico realizado en los años 2004 y 2005 (Muñoz y Vidal, 2006) se puede deducir una ordenación urbana en la que tres ejes paralelos, con una separación de aproximadamente 50 metros entre ellos, marcan tres líneas directrices. De éstas, la central corresponde con el eje de la Gran Calzada; la que está a poniente es el eje que estructura una sucesión de plazas delimitadas por montículos de escasa altura (Grupo Oeste) y, por último, la que queda al oriente es el eje principal que ordena las más grandes estructuras urbanas de La Blanca, desde la denominada Gran Plaza Norte, pasando por la Acrópolis y por la Aguada, hasta desembocar en el conjunto monumental más alejado del núcleo central, conocido como Grupo Sur (ver mapa 2).

A menos de un kilómetro de este último grupo se encuentra el río Salsipuedes, afluente del río Mopán, verdadero eje fluvial de la zona y que presumiblemente en la antigüedad fue el vertebrador de todo un sistema de comunicación comercial. Carecemos de datos fidedignos sobre la amplitud o posición de las aguas de estos ríos durante el período Clásico Tardío —600-850 d. C.- , pero es razonable pensar que las crecidas de ambos ríos anegarían amplias zonas y que sería relativamente fácil desembarcar a orillas de los numerosos asentamientos urbanos que poblaban esta región del sureste de Petén. Deducimos, por tanto, que uno de los principales accesos a La Blanca era desde el sur.

Los primeros edificios con los que habría de encontrarse el viajero que llegara a La Blanca desde el río Salsipuedes tuvieron que ser los del Grupo Sur, donde destaca la presencia de dos templos piramidales, situados en el lado oriental de la plaza principal de este conjunto - Plaza Sur - y con sus fachadas principales mirando a poniente. Estos templos gemelos y pareados parecen ser los centinelas que desde la antigüedad vieron desarrollarse la ciudad hacia el norte, ya que es en este sector donde se documentaron, hasta el momento, las fases más antiguas de ocupación del sitio, que se remontan al Clásico Temprano —250-600 d. C.—. 
A estos templos en la actualidad los denominamos 10Ll y 10L2, siendo este último el que se conserva en mejor estado. Una escalinata de casi seis metros de anchura y 18 peldaños se eleva sobre los dos cuerpos de la base piramidal hasta la plataforma donde se asienta el templo superior. Sobre esa plataforma aún se conservan los restos de los gruesos muros que conformaban un templo de algo más de nueve metros de fachada, con una estancia interior de unos 12 metros cuadrados y con un único vano central de entrada. Su coronación debió elevarse unos cinco o seis metros sobre la base superior de la pirámide, y la parte más alta alcanzaba algo más de diez metros sobre el pavimento de la citada Plaza Sur. Junto a estas pirámides, una pequeña acrópolis conforma la esquina sureste del conjunto monumental (ver foto 1 ).

Otro edificio que aún no ha sido investigado, el denominado 9Kl, delimita la longitud de esa plaza por el norte, por lo que podemos deducir que se trataba de una plaza cuadrada, de unos 3600 metros cuadrados de superficie. Observando el plano topográfico es posible comprobar que, si se compara la orientación de este conjunto monumental con los que están más al norte, hay una diferencia de seis grados sexagesimales, es decir, que su desviación desde el norte geográfico hacia el oeste es la mitad de la que tiene en la zona de ocupación más tardía - 12Nㅡ-, lo que parece corroborar nuestra hipótesis inicial de la existencia en este sector de una primitiva ocupación.

Desde el ángulo noroccidental de la Plaza Sur partía la Gran Calzada, de 35 metros de amplitud, que recorría unos 250 metros hacia el norte. Sin lugar a dudas fue éste el espacio urbano destinado a desfiles y procesiones que unía la parte más antigua del asentamiento, lugar de veneración de sus ancestros, con el centro del poder político de la nueva urbe, estructurado en torno a la Acrópolis y la Gran Plaza Norte, un nuevo centro de poder surgido en el Clásico Tardío con una función mucho más administrativa que ceremonial o religiosa en vista de las tipologías arquitectónicas que predominarán en este período.

$\mathrm{Al}$ oriente de la Calzada, entre el Grupo Sur y la Acrópolis, se encuentra una espectacular aguada, hoy desecada, que debió ocupar una superficie de unos 3000 metros cuadrados, y en la que se reflejaría la imagen de la arquitectura que coronaba la Acrópolis. En su época de apogeo, este depósito pudo llegar a conservar unos 5000 metros cúbicos de agua que, sin duda, constituía la reserva del preciado líquido en época de secas para uso de los habitantes de La Blanca. Es posible que este espacio rehundido se haya generado tras extraer la piedra utilizada para la construcción de las nuevas edificaciones del Clásico Tardío.

Muy diferente es el espacio urbano que se extiende al occidente de la Calzada - Grupo Oeste-, organizado en torno a un conjunto de plazas de gran amplitud, bordeadas por plataformas de mediana altura, sobre las que se construyeron edificios fabricados con materiales perecederos, de ahí que actualmente no se conserve ningún vestigio de estas arquitecturas. Indudablemente era en esta área residencial donde vivía la población y donde realizaba sus actividades artesanales. Los campos destinados al cultivo y a la ganadería también se encontraban en este sector (ver figura 1)

La sucesión de plazas del Grupo Oeste asciende en paralelo a la Calzada, siguiendo el eje más occidental, aunque con un ritmo más pausado aún que la suave pendiente que posee aquélla y que le permite elevarse unos nueve metros desde su salida de la Plaza Sur hasta el nivel de la Gran Plaza Norte en donde desemboca, es decir, con una pendiente inferior al $4 \%$.

Esta plaza está bordeada por el norte y poniente por dos grandes edificios que al unirse cierran la esquina noroeste de la misma. Sin embargo, hacia el oriente el espacio urbano está mucho más abierto, con una sola pequeña edificación en el centro de ese flanco. Las construcciones situadas a poniente son de carácter palaciego y de buena factura, y su trasera es un muro de sillería ciego que delimita el acceso paralelo a la plaza. El edificio del norte presenta una mayor altura y en la actualidad alcanza los diez metros sobre el nivel de la plaza; es muy posible que haya tenido alguna construcción perecedera sobre su cima ya que en ésta no se han hallado vestigios arquitectónicos.

Esta enorme plaza, que tiene como escenario principal la imponente fachada norte de la Acrópolis, 
fue sin duda el lugar para las grandes celebraciones. Con una superficie de más de 5000 metros cuadrados, se calcula que podría haber dado cabida a unas 20000 personas (ver figura 2).

La Acrópolis fue el centro del poder político y lugar de residencia de la élite. Desde la Gran Plaza Norte se accedía a ella a través de una majestuosa escalinata de 12 metros de anchura construida en la fachada norte del basamento. La escalinata avanza unos 14 metros dentro de la plaza y conserva aún sus primeros peldaños de 0,4 metros de altura perfectamente estucados (ver foto 2).

Elenorme basamento de la Acrópolis está compuesto por cuerpos aterrazados cuyo interior encierra edificios - subestructuras - de una época anterior. Este basamento está coronado por un palacio de grandes dimensiones en forma de C - Palacio 6J2-, que conforma un patio central de 36 metros de lado, abierto hacia el oriente. Ese flanco está ocupado por otro palacio - 6J1- al que hemos denominado Palacio de Oriente (Muñoz, 2007). De forma rectangular, posee unos 28 metros de fachada, dejando en los laterales norte y sur del mismo dos pasos de entrada al patio central. Este edificio abre sus tres puertas hacia ese patio, siendo la central la de mayor tamaño y la que da acceso a lo que debió de ser la sala de audiencias del dirigente de La Blanca, una sala que se eleva dos metros sobre el pavimento del patio mediante una escalinata de acceso y que presenta unas proporciones inusuales en el ámbito de la arquitectura maya, capaz de albergar en su interior a más de 100 personas (ver figura 3).

Gracias a las escenas de corte plasmadas en las cerámicas polícromas sabemos que los encuentros políticos o de otra naturaleza que tenían lugar en el interior de esas estancias eran contemplados por otros individuos, concentrados, seguramente, en el amplio patio sobre el que volcaba su fachada este palacio y en las propias escalinatas que conducían a su interior, como si de un espectáculo teatral se tratara (Houston e Inomata, 2009: 115). Los hermosos grafitos plasmados en el muro trasero de la gran sala central reflejan escenas de este tipo que muy bien pudieron haber ocurrido en ese mismo espacio (Vidal y Muñoz, 2009: 109-118).
En consonancia con sus proporciones en planta, las claves de su bóveda se elevan más de seis metros sobre el pavimento actual, el cual fue recrecido al menos 1,5 metros por encima del pavimento original. Esta gran estancia posee dos pequeñas salas laterales más privadas a las que se accede por sendas puertas situadas en las esquinas del lado occidental y en cuyo interior se encuentra una banqueta que ocupa más de la mitad oriental de cada una de las salas. La que está situada al sur conserva casi la totalidad de su bóveda y en ella se puede apreciar la calidad y la finura de los trazados de estereotomía y del corte geométrico de las dovelas, especialmente en las situaciones de encuentro, en las esquinas entre la bóveda y el muro testero. En ese lugar los canteros mayas tuvieron que trazar y labrar unas dovelas cuyas caras debían presentar las dos diferentes inclinaciones que tenía la propia bóveda y el muro testero, el cual, aún siendo inclinado, trabaja y traba como un muro de sillería (Muñoz, Vidal y Perelló, 2008). Según nuestra hipótesis, esas salas contiguas servían como lugar de almacenamiento de la parafernalia ritual y de los tributos que eran entregados a los dirigentes durante el transcurso de pomposas celebraciones.

Las otras dos salas que flanquean los extremos norte y sur del palacio abren también sus puertas hacia el patio de la Acrópolis y tienen una inusual dimensión, ya que son de planta casi cuadrada de más de 16 metros cuadrados y están cubiertas por bóvedas de lados equivalentes, una rara tipología arquitectónica muy poco frecuente en la arquitectura maya. En definitiva, esta estructura arquitectónica de cuidada sillería supone un verdadero alarde estructural en el área maya.

El enorme palacio que circunda por tres lados la Acrópolis -6J2- tiene unas características formales muy elaboradas; sus tres fachadas han sido compuestas siguiendo el mismo orden minucioso, con nueve puertas en cada una de ellas que dan acceso a diferentes estancias, de las que solamente las centrales son pasantes para permitir los accesos al patio central. Las puertas de estos cuartos centrales son de mayor amplitud que las restantes, aunque todas tienen una misma altura de dinteles de aproximadamente cuatro metros sobre el piso, una dimensión que casi duplica la 
habitual de las construcciones palaciegas de la zona. Las estancias que posee este edificio, excepto las centrales, cuya función, como ya se ha dicho, era de paso, son de doble puerta y disponen de amplias banquetas interiores que ocupan gran parte de la superficie de la sala. Por la altura de las claves de sus bóvedas, puede decirse que nos encontramos ante edificios palaciegos de muy buena factura arquitectónica y de dimensiones colosales en relación con las habituales tipologías del área maya.

El eje norte-sur de este conjunto arquitectónico está claramente marcado por dos puertas enfrentadas en el patio, con sus correspondientes escalinatas monumentales, que facilitan el acceso a la Gran Plaza por el norte y permiten la salida hacia la terraza sur, la cual presenta vistas hacia la Aguada y el Grupo Sur. En la parte meridional se le anexionó un cuarto alargado con tres puertas mirando al norte y una pequeña puerta a modo de pasaje, cubierta con una pequeña bóveda de aproximación que da paso hacia el este, en dirección al acceso que deja por el sur el Palacio de Oriente.

En la terraza sur, un palacio de menores dimensiones, con tres puertas en su fachada principal y arquitectura más tardía - Palacio 6J3-, cierra el flanco oeste del Palacio 6J2. Posee dos crujías, la segunda de las cuales presenta una distribución muy parecida tipológicamente a la del Palacio de Oriente, pero a menor escala. La constatación hecha por las investigaciones de que en sus muros se utilizó el cinabrio como pigmento, al igual que en el Palacio de Oriente, lleva a pensar que posiblemente este palacio fue ocupado por algún miembro destacado de la familia del gobernante.

Así, desde la Calzada, el frente oeste de la Acrópolis se presenta como una sucesión de tres cuerpos ataludados, desde donde arrancaba una imponente fachada de 50 metros de longitud y nueve metros de altura, jalonada de forma rítmica por 13 enormes puertas, todas de igual amplitud menos la central, que tenía mayor anchura, lo que denotaba que era el acceso principal hacia los espacios interiores de la Acrópolis. Tanto esta puerta como las centrales de las fachadas norte y sur tienen dispuestas banquetas a ambos lados.
En su conjunto, los monumentales muros de sillería de los palacios de la Acrópolis presentan un aspecto de fortaleza que fue el motivo de que el sitio fuera bautizado en 1905 por uno de sus primeros visitantes científicos, el investigador y aventurero austríaco Teobert Maler, con el sobrenombre de "El Castillito" (Quintana, 2005: 21), en referencia a la potente arquitectura que asomaba sobre los montículos. De hecho, el estudio del sistema constructivo de estas edificaciones nos ha inclinado a pensar que el centro del noreste al que estaba asociada La Blanca era Nakum, debido a que sus muros de sillería tienen unos sistemas constructivos y proporciones parecidos, y algunas de las tipologías arquitectónicas pueden presentar ciertas similitudes, si bien aún no podemos aportar datos concluyentes desde el momento en que carecemos de otros indicadores - arqueológicos, epigráficos o iconográficos - que nos permitan reforzar esta hipótesis.

Además, en la antigüedad estos edificios estaban estucados y pintados de vivos colores, y sus frisos se encontraban decorados con relieves de los que apenas se han conservado algunos ejemplares. Si pensamos que estos palacios se elevaban hasta los 16 metros por encima del pavimento de la plaza que, como ya se ha dicho, podía albergar a varios miles de personas, no es difícil imaginar la impresión que hubieron de causar a todo aquel que llegara a La Blanca, pues precisamente ése debió ser el propósito de sus promotores y constructores: crear un espacio en el que reunir a todos los miembros de las comunidades y transmitir, a través de la potencia de la arquitectura, valores y principios ideológicos estrechamente ligados a la élite.

En definitiva, los edificios que predominan en La Blanca son de tipo residencial o representativo - palacios-, destacando la ausencia de conjuntos monumentales para celebraciones rituales tales como los conjuntos de tipo Grupo E, conjuntos de Acrópolis con complejos tríadicos, terrenos para el Juego de Pelota y conjuntos de monumentos tallados. Nuestra hipótesis es, por tanto, que durante el período Clásico este centro urbano detentó funciones de índole civil o administrativa, relacionadas con actividades propias de un centro de frontera, tales como intercambio 
de productos o recepción de tributos, vinculadas tanto a sitios del noreste de Petén como de Belice y del sureste petenero - Montañas Mayas- (Vidal y Muñoz, 2011).

Estas funciones de carácter administrativo debieron desarrollarse en los palacios de la Acrópolis, especialmente en el Palacio de Oriente, y en los que coronaban los basamentos que delimitan la Gran Plaza Norte.

La creciente prosperidad económica habría sido la causante de que en los albores del Clásico Terminal — 850-1000 d. C.- estos edificios fueran objeto de profundas remodelaciones de carácter constructivo, seguramente con la intención de aumentar su tamaño y monumentalidad. Fue entonces cuando se emplearon pigmentos de importación, como el cinabrio, para pintar los muros de sus edificios principales (Vázquez, Vidal y Muñoz, 2013). En el área maya, la presencia de cinabrio es habitual en contextos funerarios, pero su uso con fines no ceremoniales debió ser muy restringido, lo que denota la importancia de este sitio en esos años.

Pero su auge económico rápidamente se vio afectado por la profunda crisis que a finales del siglo IX azotó Petén, con nefastos efectos en las rutas comerciales que antaño tantos beneficios habían otorgado a los centros urbanos de esta región de la cuenca del río Mopán. La Blanca fue uno de esos centros y las huellas de su precipitado abandono por parte de la élite dirigente han quedado reflejadas en la interrupción de las remodelaciones que por aquel entonces se estaban llevando a cabo en todos sus conjuntos monumentales.

\section{La crisis final del Clásico Tardío en La Blanca}

Durante ese convulso período, el panorama de todos estos territorios de las tierras bajas mayas del sur debió ser desolador, con grupos humanos desplazándose de un sitio a otro, tanto los miembros dela élite como las poblaciones campesinas. ¿A dónde emigró la élite dirigente de La Blanca? No lo sabemos pero sí se ha podido documentar una reocupación del sitio en esos años.
Estos nuevos habitantes se instalaron en los palacios de la Acrópolis, donde llevaron a cabo actividades domésticas muy alejadas de aquéllas realizadas por sus primitivos ocupantes. La presencia de basureros en el patio de la Acrópolis y el estudio de su contenido son testimonios de que estos nuevos moradores no pertenecían a la élite y de que se instalaron en las dependencias palaciegas sin ocuparse de su adecuado mantenimiento, mientras que el resto de los edificios de La Blanca se quedaron ya para siempre a medio remodelar. La situación política del momento debió ser francamente inquietante, de ahí que se mantuvieron en cierta forma atrincherados en la Acrópolis, como así lo demuestra el hecho de que algunos de sus accesos fueran clausurados (Vidal y Muñoz, 2011).

Esta situación no habría de durar mucho tiempo y el definitivo abandono del sitio en tiempos del Clásico Terminal se produjo de forma violenta. El hallazgo de más de una decena de enterramientos sobre superficie en diferentes espacios de este conjunto monumental - patio interior, terrazas del lado sur, interior de los cuartos - constituye un claro ejemplo de cómo la crisis del Clásico Terminal afectó a estos sitios de la cuenca del río Mopán, ya que estos individuos, en vez de haber sido debidamente inhumados según el ritual maya tradicional, fueron dejados expuestos sobre el nivel del piso, y algunos de ellos presentaban signos de violencia (Valdés y Vidal, 2007: 177-179) (ver figura 4).

A partir de entonces, los edificios abandonados fueron víctimas de las agresiones medioambientales y del paso del tiempo, de modo que acabaron parcialmente colapsados sobre espesas capas de derrumbe.

Y asílos encontraron unos 100 años más tarde grupos humanos del período Postclásico Temprano (1000-1200 d.C.) que, de forma esporádica, se instalaron en estas dependencias parcialmente destruidas. En ellas dejaron a modo de ofrenda grandes ollas depositadas boca abajo y otros restos de cultura material, e incluso algunos encontraron sobre el escombro su lugar de descanso eterno, pues el estudio de los enterramientos hallados en esos niveles ha demostrado que pertenecen a ese período. 
Tras esas esporádicas ocupaciones de época postclásica, La Blanca permaneció parcialmente sepultada por la espesa vegetación, si bien existe constancia de la presencia de exploradores y otros curiosos que, al menos desde el siglo XVIII, comenzaron a visitar el sitio y dejaron su firma en los muros de los palacios semidestruidos; por ello, estos grafitos de época moderna se encuentran a la altura del arranque de las bóvedas de estos edificios, únicas partes visibles de los mismos (Muñoz, Vidal y Haeussler, 2009).

Más adelante, a comienzos del siglo XX, el investigador estadounidense Raymond E. Merwin hizo interesantes fotografías de las arquitecturas visibles del sitio, las cuales fueron de gran ayuda para la formulación del Proyecto La Blanca.

\section{La conservación del patrimonio cultural y las acciones de desarrollo sostenible del Proyecto La Blanca}

El Proyecto arqueológico La Blanca surgió como una propuesta conjunta de la Universidad de Valencia y de la Universidad Politécnica de Valencia, apoyadas por la Universidad de San Carlos de Guatemala, y fue financiado por el Ministerio de Cultura de España dentro de su Programa de Excavaciones en el Exterior. El objetivo principal era la investigación y puesta en valor del asentamiento urbano de La Blanca, que había sido identificado, como decíamos, desde inicios del siglo XX, pero en el que nunca se había realizado una profunda investigación arqueológica y arquitectónica.

Otro de los objetivos de este proyecto era la puesta en marcha de diferentes actuaciones conducentes al desarrollo social y económico de las comunidades del entorno del sitio arqueológico, especialmente de la aldea de igual nombre localizada a menos de dos kilómetros de distancia, con el convencimiento de que actualmente ya no se puede hablar aisladamente de patrimonio cultural o de investigación científica sin que todo esto conlleve un compromiso ético y social con las poblaciones en donde están enclavados estos bienes culturales.
La aldea La Blanca tenía una población cercana a los 1000 habitantes en 2004 (Monterde, 2004: 164) y se creó en el proceso de colonización que el Gobierno de Guatemala llevó a cabo hace unos 50 años, mediante el traslado de familias de otros departamentos y la adjudicación de tierras en esta zona de Petén. La población actual de La Blanca se dedica principalmente a la agricultura y a la ganadería, y su asentamiento carece de algunas infraestructuras básicas tales como sistema de evacuación de aguas y electricidad. Posee un sistema propio de distribución primaria de agua desde unos depósitos que se abastecen de dos manantiales subterráneos naturales.

Las viviendas son en general autoconstruidas y de carácter popular, edificadas a partir de los materiales disponibles, normalmente madera o bajareque, con cubiertas vegetales, aunque también hay algunas realizadas con fábricas más sólidas, edificadas con bloques de cemento y cubiertas de lámina metálica.

Como decíamos, el Proyecto La Blanca se concibió desde un principio como un proyecto de patrimonio cultural con una clara visión de desarrollo de las poblaciones vinculadas. En este sentido, desde un principio se realizó un estudio de caracterización social, económica y cultural de la población de La Blanca para programar una serie de acciones de concienciación y formación que posibilitaran que la actuación de investigación científica, recuperación, restauración y puesta en valor del sitio arqueológico constituyeran a la vez un motor de desarrollo social, económico y cultural de los habitantes aledaños.

Dichas acciones se desarrollaron de forma ininterrumpida desde el año 2005 hasta el 2012, y la implicación de los habitantes de La Blanca en el proyecto ha sido muy intensa desde sus principios. Cada temporada de campo, entre 25 y 30 personas de la población trabajan y se forman en las técnicas de excavación y conservación, con las que adquieren en algunos casos gran capacidad y destreza. De forma paralela, se ha fomentado el conocimiento del sitio arqueológico y del área maya en general mediante talleres de sensibilización en la protección y conservación del patrimonio cultural (Vidal, Muñoz, 
Vázquez y Horcajada, 2010), y con el apoyo del Instituto Guatemalteco de Turismo se han ofrecido cursos de capacitación de guías y anfitriones comunitarios en los que los asistentes recibieron formación para recibir a visitantes y explicarles tanto las características y valores culturales del sitio arqueológico, como las costumbres y modos sociales de la población de La Blanca (ver foto 3).

La construcción de un centro de interpretación a la entrada del sitio arqueológico (Vidal y Muñoz, 2008; Muñoz, Vidal, Martín y Peiró, 2010), así como el diseño de senderos interpretativos y de paneles informativos en el interior del mismo, han fomentado en gran medida la afluencia de turistas así como la difusión de los trabajos de investigación y puesta en valor de este antiguo asentamiento maya.

La puesta en marcha en el año 2012 del taller de teatro "La Blanca a escena", protagonizado por la población infantil de La Blanca, ha contribuido a estrechar los lazos de identidad de este sector de la población, tanto con el pasado maya como con las ruinas arqueológicas, algo fundamental para que las valoren y se sientan portadores y responsables de este rico patrimonio cultural y natural.

En definitiva, todas estas acciones han sido diseñadas con la idea de convertirse en un programa de referencia para otros muchos sitios arqueológicos vinculados a pequeñas poblaciones en el área de Petén en los que es factible realizar proyectos de patrimonio cultural y desarrollo sostenible similares, creando así una red territorial destinada a favorecer un turismo cultural alternativo de baja intensidad. Un turismo susceptible de suponer una mejora en las condiciones sociales y económicas de estas poblaciones, poseedoras de un extraordinario patrimonio cultural.

\section{Epílogo}

Con este proyecto se ha podido demostrar cómo, mediante las intervenciones realizadas por instituciones científicas de investigación dirigidas a la excavación, estudio y puesta en valor del patrimonio cultural maya, se puede, al mismo tiempo, realizar acciones susceptibles de favorecer el desarrollo social, económico y cultural de las poblaciones vinculadas territorialmente a estos bienes patrimoniales.

El Proyecto La Blanca ha tenido, desde sus orígenes, este doble planteamiento en el convencimiento de que en la situación actual de países como Guatemala no es posible programar acciones deinversión en investigación del patrimonio sin considerar forzosamente cómo repercuten e inciden en el desarrollo.

Por otro lado, la contribución que se ha realizado, tras estos años de investigación, al conocimiento de cómo fue y qué papel jugó esta antigua población de La Blanca en el período histórico en que alcanzó su esplendor, y en el proceso de crisis y decadencia posterior, sin duda ha aportado nuevas claves que permiten comprender mejor este tan debatido período histórico del Clásico Terminal en las tierras bajas mayas del Petén guatemalteco, si bien es preciso continuar con las investigaciones en el resto de las entidades políticas de esta región con el fin de llegar a comprender la complejidad interna de la misma y de las relaciones que estos centros mantuvieron entre sí.

\section{Notas}

${ }^{1}$ Los autores agradecen expresamente el apoyo del Ministerio de Economía y Competitividad de España a través de la financiación de los proyectos de investigación coordinados con números de referencia BIA201l28311-C02-01 y 02, así como el patrocinio del Ministerio de Educación, Cultura y Deportes de España a través de la financiación obtenida por el Proyecto Arqueológico La Blanca dentro del programa de ayudas para Proyectos Arqueológicos en el Exterior. Asimismo, la Agencia Española de Cooperación Internacional y el Ministerio de Cultura y Deportes de Guatemala contribuyeron de forma determinante a hacer posibles las investigaciones y la obtención de los resultados que se exponen en esta publicación.

\section{Referencias bibliográficas}

Ciudad Ruiz, Andrés y María Josefa Iglesias Ponce de León (2001), "Un mundo ordenado: la ciudad 
maya y el urbanismo en las sociedades antiguas", en Andrés Ciudad Ruiz, María Josefa Iglesias Ponce de León y María del Carmen Martínez Martínez (eds.) Reconstruyendo la ciudad maya: el urbanismo en las sociedades antiguas, Madrid: SEEM, pp. 11-40.

Houston, Stephen D. y Takeshi Inomata (2009), The Classic Maya, Cambridge: Cambridge University Press.

Laporte Molina, Juan Pedro (1996), “Organización territorial y política prehispánica en el sureste de Petén”, en Atlas Arqueológico de Guatemala, núm. 4, Guatemala: Instituto de Antropología e Historia.

Laporte Molina, Juan Pedro (1998), "Una perspectiva del desarrollo cultural prehispánico en el sureste de Petén”, en Andrés Ciudad Ruiz, Yolanda Fernández Marquínez, José Miguel García Campillo, María Josefa Iglesias Ponce de León, Alfonso Lacadena García Gallo y Luis T. Sanz Castro (eds.), Anatomía de una civilización: aproximaciones interdisciplinares a la culturamaya, Madrid: SEEM, pp. 131-160.

Laporte Molina, Juan Pedro (2001), "Dispersión y estructura de las ciudades del Sureste de Petén, Guatemala”, en Andrés Ciudad Ruiz, María Josefa Iglesias Ponce de León y María del Carmen Martínez Martínez (eds.), Reconstruyendo la ciudad maya: el urbanismo en las sociedades antiguas, Madrid: SEEM, pp. 137-161.

Laporte Molina, Juan Pedro y Héctor E. Mejía (2001), “Los sitios arqueológicos de la cuenca del río Salsipuedes en el Sureste de Petén, Guatemala”, en Mexicon, vol. XXIII, núm. 3, pp. 65-72.

Monterde Díaz, Rafael (2005), “La Blanca: Una iniciativa piloto de incidencia de la recuperación del patrimonio maya en el desarrollo local", en Gaspar Muñoz Cosme y Cristina Vidal Lorenzo (eds.), La Blanca. Arqueologíay desarrollo, Valencia: Editorial UPV, pp. 161-173.

Muñoz Cosme, Gaspar (2007), "El Palacio de Oriente", en Cristina Vidal Lorenzo y Gaspar Muñoz Cosme (eds.), La Blanca y su entorno. Cuadernos de arquitectura y arqueología maya, Valencia: Editorial UPV, pp. 2l-28.

Muñoz Cosme, Gaspar y Cristina Vidal Lorenzo (eds.) (2005), La Blanca. Arqueología y desarrollo, Valencia: Editorial UPV.

Muñoz Cosme, Gaspar y Cristina Vidal Lorenzo (eds.) (2006), La Blanca. Arquitectura y clasicismo, Valencia: Editorial UPV.
Muñoz Cosme, Gaspar, Cristina Vidal Lorenzo y Ricardo Perelló Roso (2008), "Características formales y constructivas de la bóveda maya del Palacio de Oriente de La Blanca", en Arché, núm. 3, pp. 335-340.

Muñoz Cosme, Gaspar, Cristina Vidal Lorenzo y Óscar Haeussler Paredes (2009), "Un testimonio de época colonial en un palacio maya. El grafito de Pedro Montañés en La Blanca", en Cristina Vidal Lorenzo y Gaspar Muñoz Cosme (eds.), Los grafitos mayas. Cuadernos de arquitectura y arqueología maya 2, Valencia: Editorial UPV, pp. 173-187.

Muñoz Cosme, Gaspar, Cristina Vidal Lorenzo, Beatriz Martín Domínguez y Andrea Peiró Vitoria (2010), "El Centro de Interpretación de La Blanca como generador de procesos de formación y capacitación en patrimonio cultural", en $V$ Seminario Internacional Cultura y Cooperación. Formación y capacitación en Patrimonio como alternativa de desarrollo sostenible, Granada: Restauradores sin Fronteras, pp. 57-60.

Quintana Samayoa, Óscar (2005), "La cuenca baja del río Mopán (Petén, Guatemala) y su patrimonio cultural maya", en Gaspar Muñoz Cosme y Cristina Vidal Lorenzo (eds.), La Blanca. Arqueología y desarrollo, Valencia: Editorial UPV, pp. 17-24.

Taladoire, Eric (2008), "From terminology to concepts. The role of vocabulary in our understanding Precolumbian Urbanism", en W. Sanders, A. G. Mastache y A. García Cook (eds.), El urbanismo en Mesoamérica/Urbanism in Mesoamerica, vol. 2, México/ Philadelphia: Instituto Nacional de Antropología e Historia/Pennsylvania State University, pp. 621-650.

Valdés Gómez, Juan Antonio y Cristina Vidal Lorenzo (2007), "Observaciones sobre el colapso y el período Clásico Terminal", en Cristina Vidal Lorenzo y Gaspar Muñoz Cosme (eds.), La Blanca y su entorno. Cuadernos de arquitectura y arqueología maya, Valencia: Editorial UPV, pp. 173-179.

Vázquez de Ágredos, María Luisa, Cristina Vidal Lorenzo y Gaspar Muñoz Cosme (2012), "Pigmentos locales e importados en la decoración mural de los palacios de la Acrópolis de La Blanca: caracterización científica e interpretación", en Adrián Velázquez Castro y Lynneth S. Lowe (eds.), Técnicas analíticas aplicadas a la 
caracterización y producción de materiales arqueológicos en el área maya, México: UNAM, pp.ll-29.

Vidal Lorenzo, Cristina y Gaspar Muñoz Cosme (2009), "Los grafitos de La Blanca. Metodología para su estudio y análisis iconográfica", en Cristina Vidal Lorenzo y Gaspar Muñoz Cosme (eds.), Los grafitos mayas. Cuadernos de arquitectura y arqueología maya 2, Valencia: Editorial UPV, pp. 99-118.

Vidal Lorenzo, Cristina y Gaspar Muñoz Cosme (2011), "La crisis de La Blanca en el Clásico Terminal", ponencia presentada en el Coloquio Sociedades Mayas Milenarias. Crisis del pasado y resiliencia. Museo del QuaiBranly, París, 1 y 2 de julio.

Vidal Lorenzo, Cristina y Gaspar Muñoz Cosme (eds.) (2007), La Blanca y su entorno. Cuadernos de arquitecturay arqueología maya, Valencia: Editorial UPV.
Vidal Lorenzo, Cristina, Gaspar Muñoz Cosme, María Luisa Vázquez de Ágredos y Patricia Horcajada Campos (2010), "Conociendo su pasado. El Programa de sensibilización en la conservación del patrimonio cultural en la comunidad maya de La Blanca (Guatemala), en $V$ Seminario Internacional Cultura y Cooperación. Formación y capacitación en Patrimonio como alternativa de desarrollo sostenible, Granada: Restauradores sin Fronteras, pp. 119-122.

Vidal Lorenzo, Cristina y María Ángeles Muñoz Cosme (2008), "Los centros de interpretación como propuesta de desarrollo cultural y social: el caso de La Blanca (Guatemala), en Gaspar Muñoz Cosme y Cristina Vidal Lorenzo (eds.), III Congreso Internacional dePatrimonio Culturaly Cooperaciónal Desarrollo, México: Museo Nacional de Antropología-AECID, pp. 77-84. 


\section{Figura 1. Reconstrucción ideal del Grupo Oeste y de la Acrópolis}

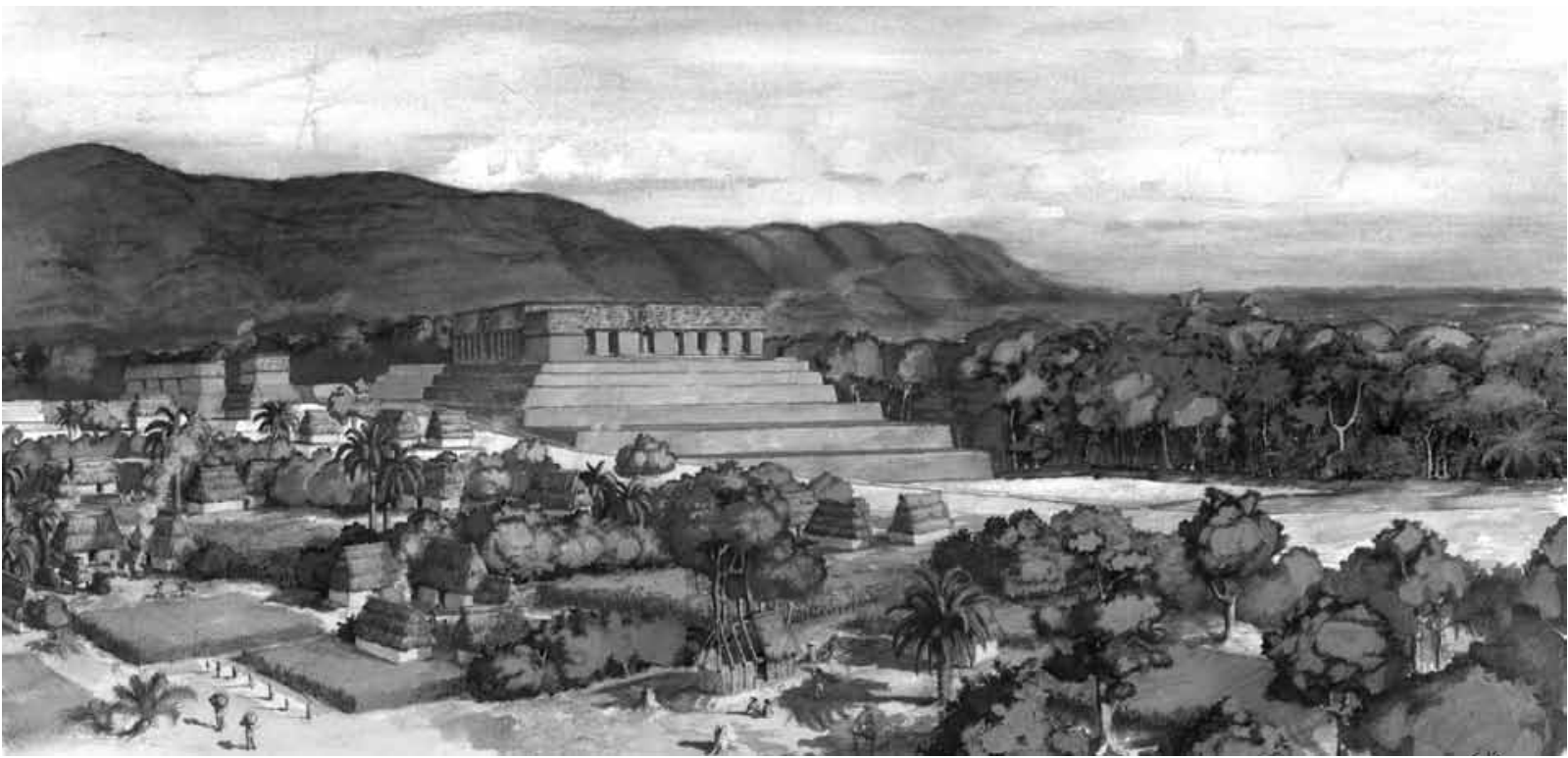

Fuente: E. Meijide.

Figura 2. Reconstrucción ideal de la Gran Plaza Norte y de la Acrópolis. Vista desde el noroeste

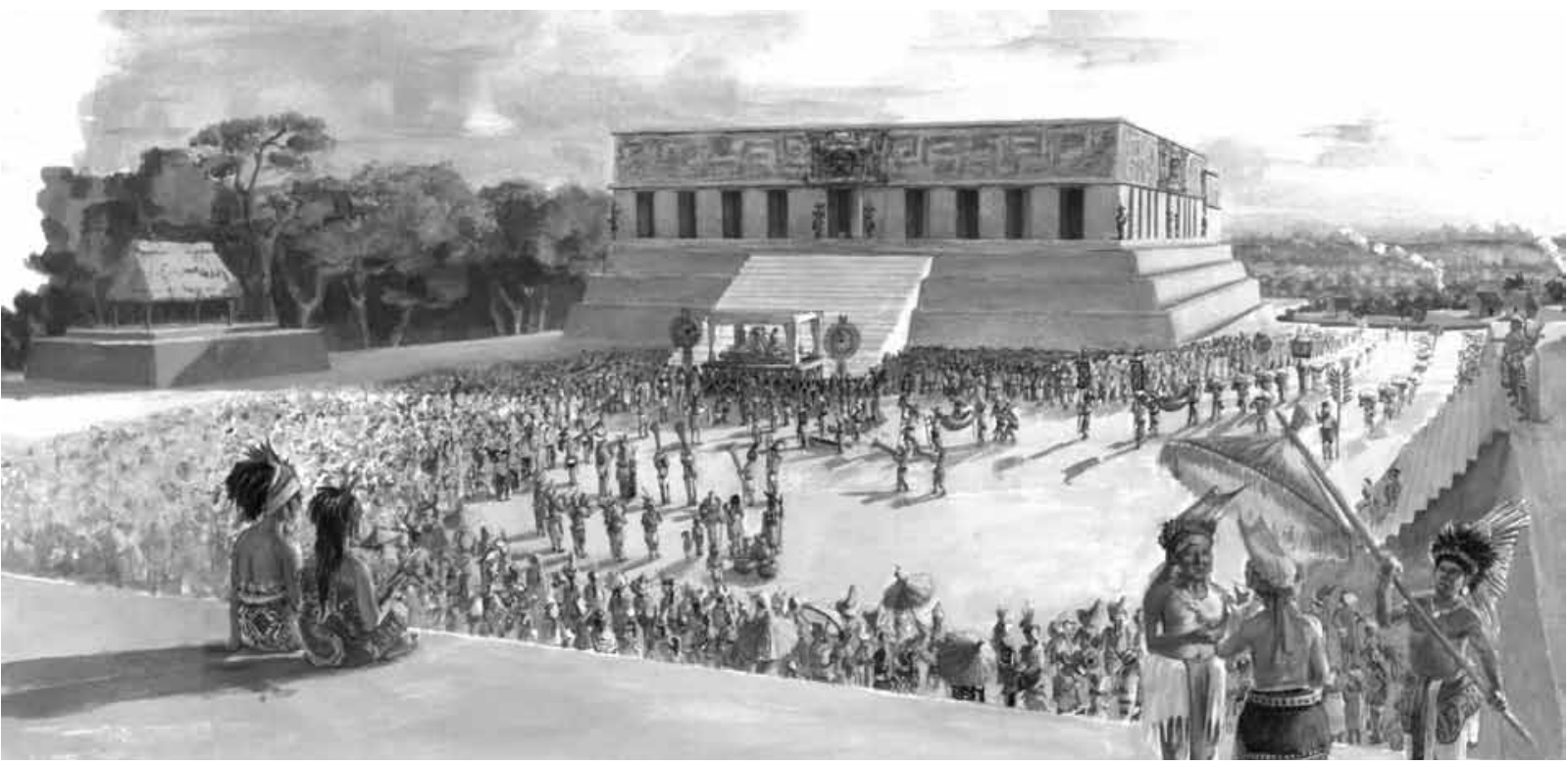

Fuente: E. Meijide. 
Figura 3. Planta y sección del Palacio de Oriente
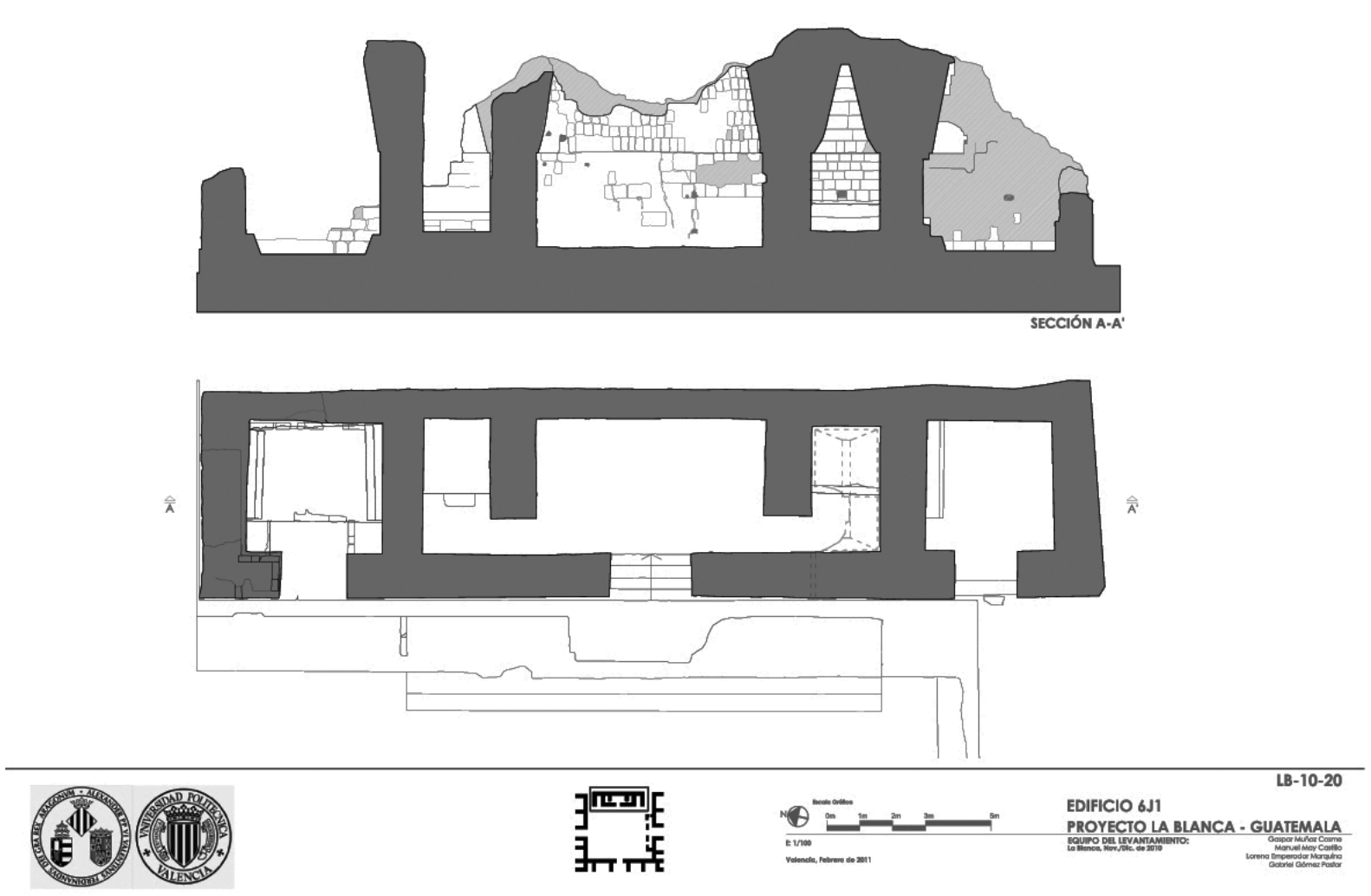

Fuente: Proyecto La Blanca, 2010. 
Figura 4. Reconstrucción ideal del Enterramiento 3, hallado en la entrada al cuarto central del Palacio de Oriente. Planta y alzado

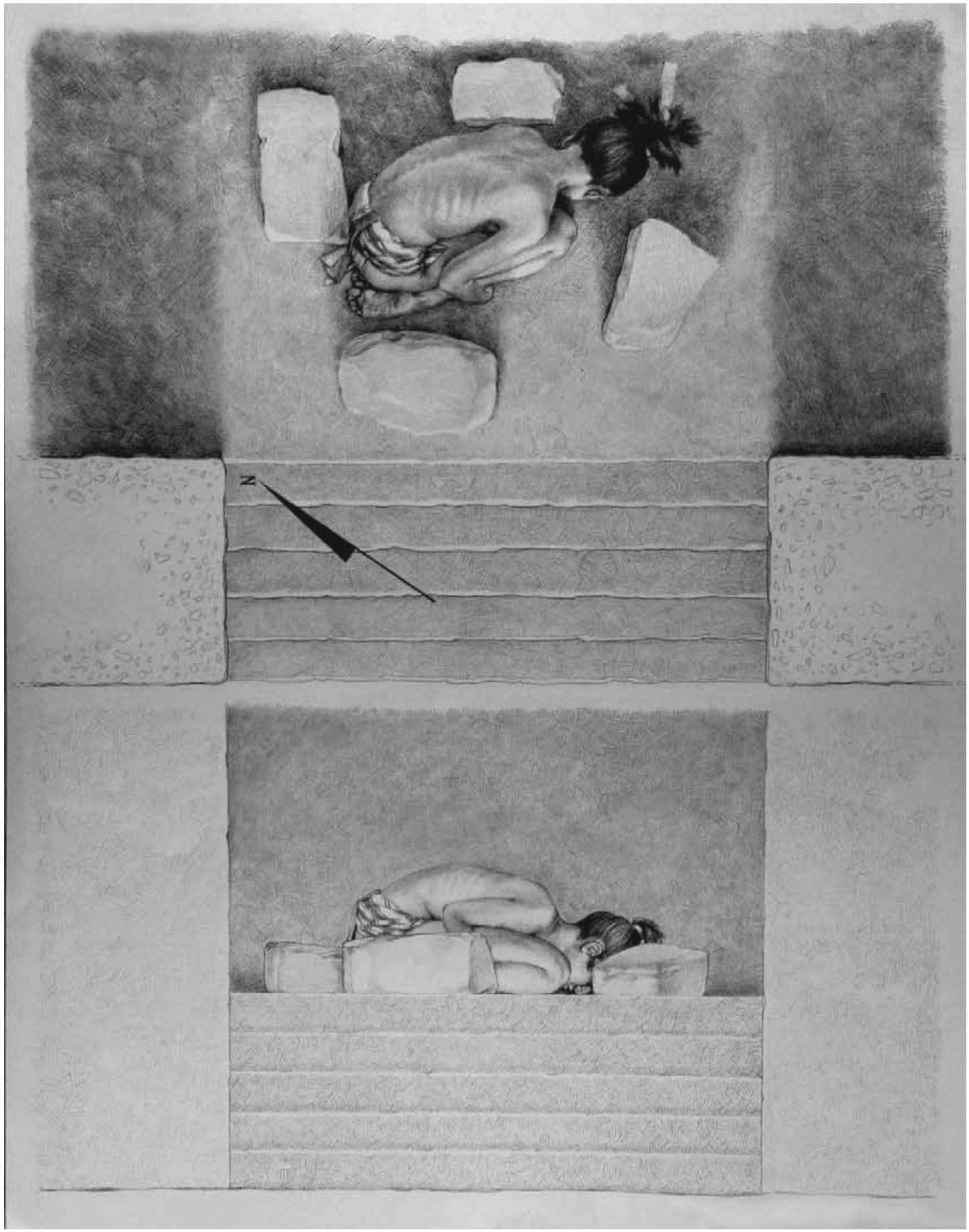

Fuente: E. Meijide. 
Foto 1. Templo piramidal 10L2 durante su excavación

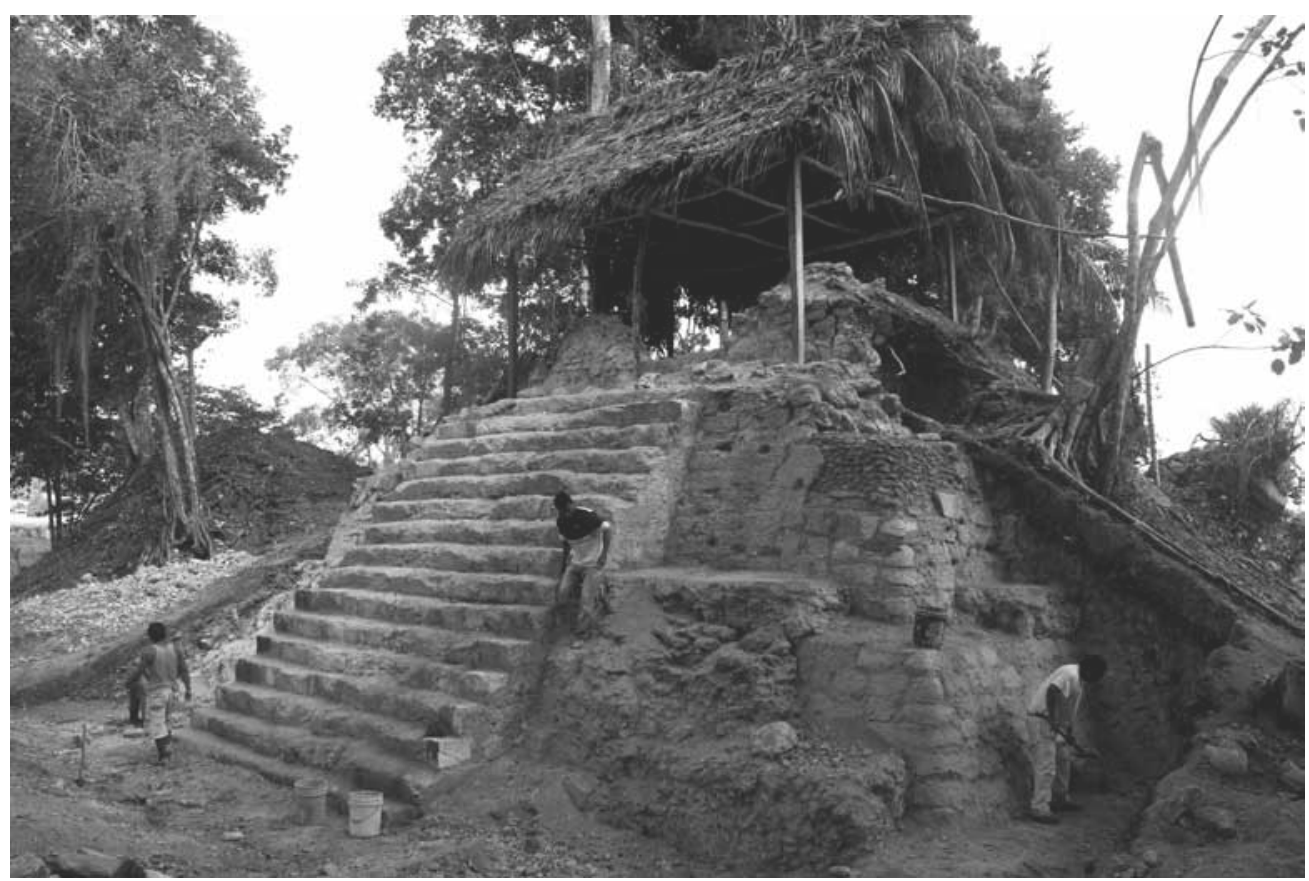

Fuente: G. Muñoz.

Foto 2. Hallazgo de la escalinata de acceso a la Acrópolis desde la Gran Plaza Norte

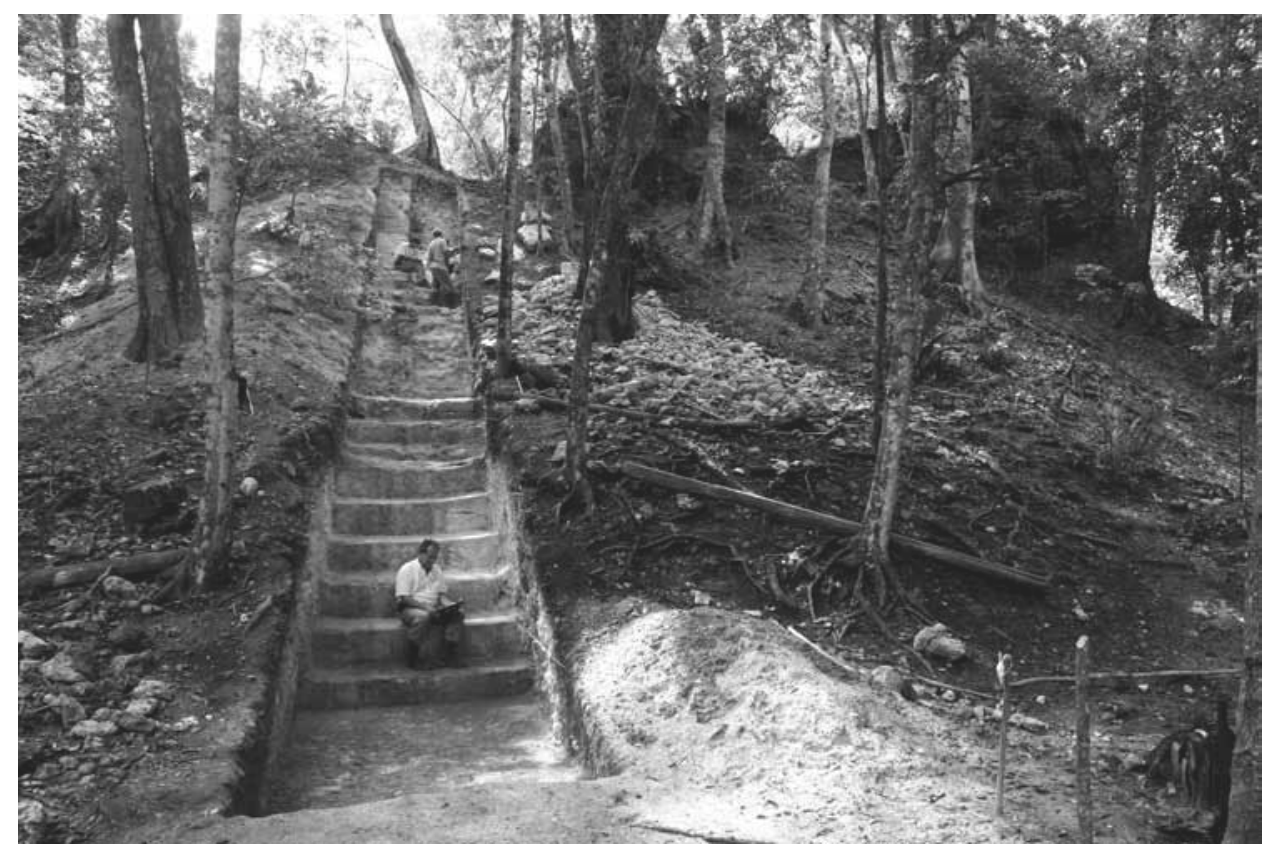

Fuente: A. Toepke. 
Foto 3. Interior del centro de interpretación de La Blanca durante la impartición de uno de los cursos de sensibilización en la protección del patrimonio cultural

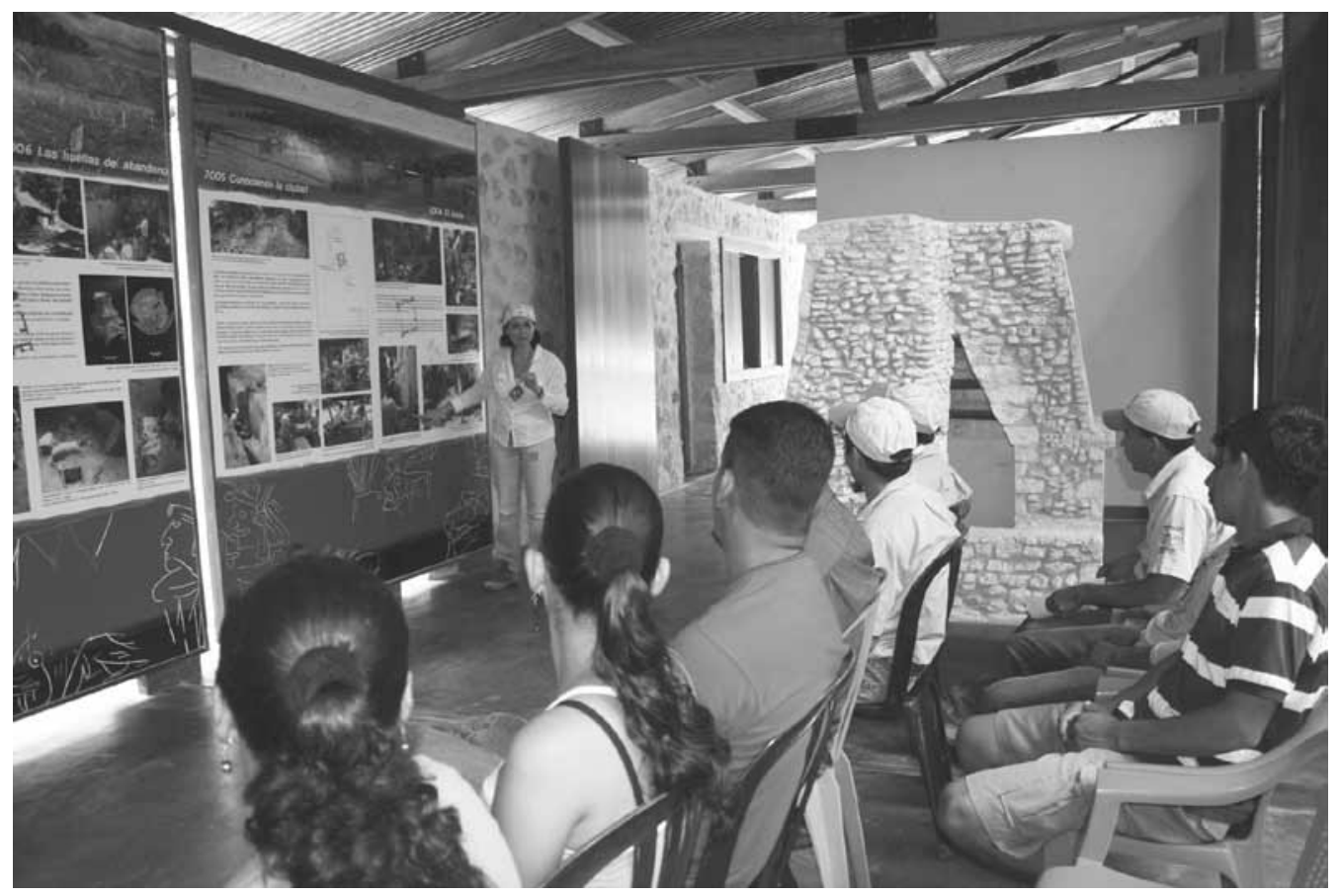

Fuente: L. Emperador.

Mapa 1. Valle del Mopán con indicación de los principales sitios arqueológicos

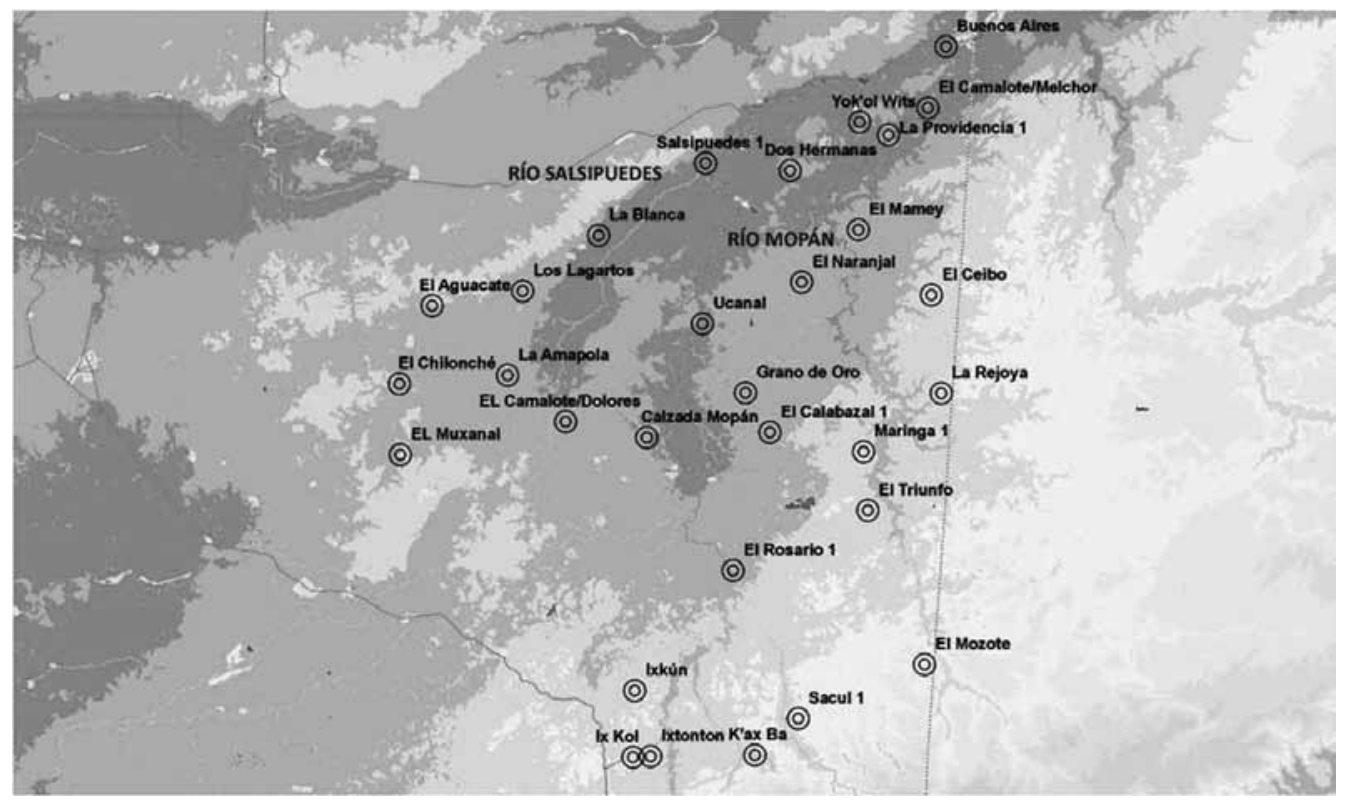

Fuente: Proyecto La Blanca, 2013. 
Mapa 2. Plano de La Blanca con indicación de sus principales conjuntos monumentales y espacios urbanos

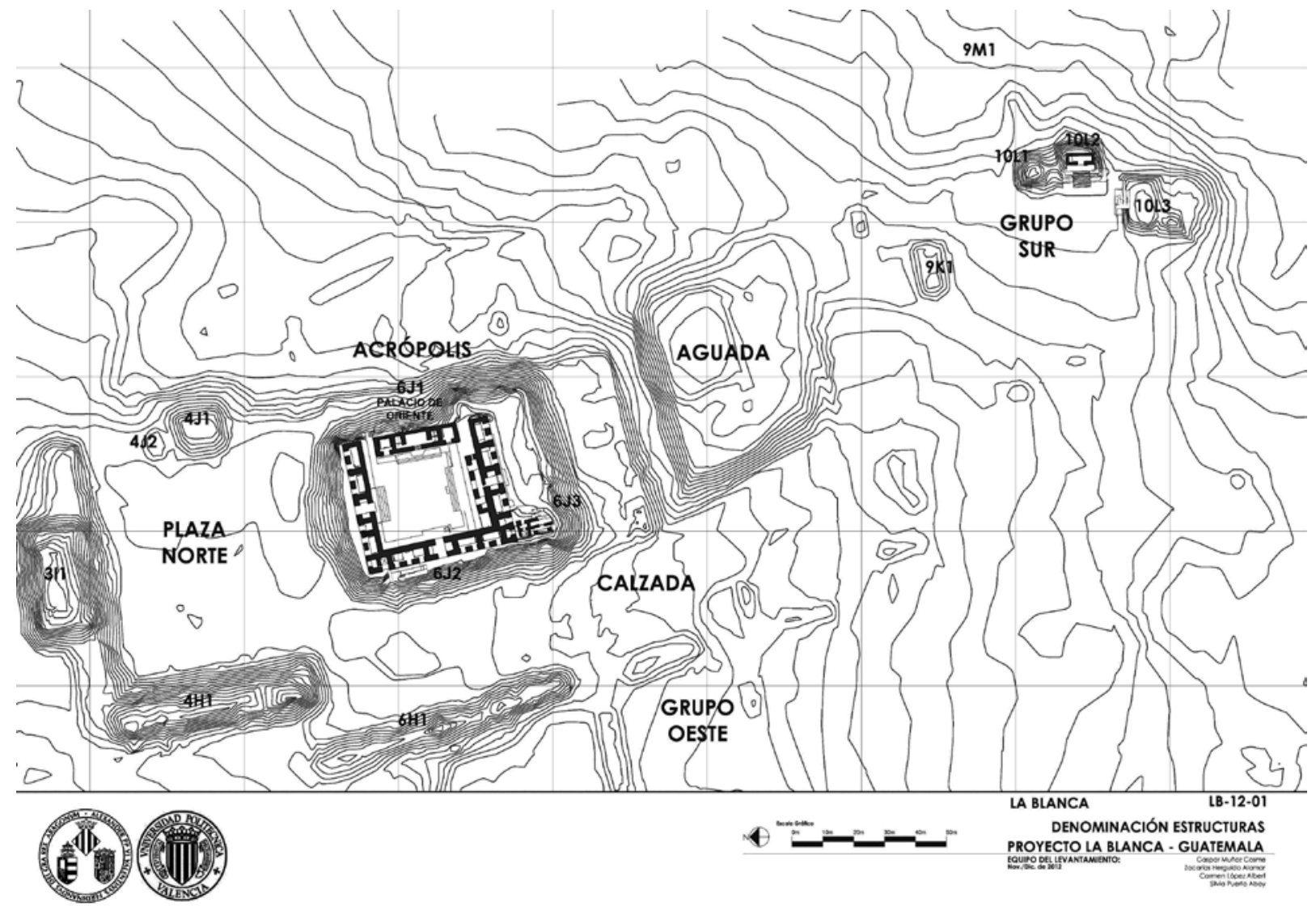

Fuente: Proyecto La Blanca, 2012. 